\title{
Epidemiology of Amoebas in Marrakech (Morocco): Experience of Parasitology Service from the Military Hospital Avicenne
}

\author{
Mouayche Ikhlas*, Moutaj Redouane, El Mezouari El Mostafa \\ Department of Parasitology and Mycology, Military Hospital Avicenne Marrakech, Faculty of medicine and Pharmacy, Marrakech, Morocco
}

Email address:

I.Mouayche@gmail.com (M. Ikhlas)

${ }^{*}$ Corresponding author

\section{To cite this article:}

Mouayche Ikhlas, Moutaj Redouane, El Mezouari El Mostafa. Epidemiology of Amoebas in Marrakech (Morocco): Experience of Parasitology Service from the Military Hospital Avicenne. Pathology and Laboratory Medicine. Vol. 2, No. 1, 2018, pp. 25-29. doi: $10.11648 /$ j.plm.20180201.15

Received: August 15, 2018; Accepted: September 5, 2018; Published: September 27, 2018

\begin{abstract}
Amoebiasis is the third leading cause of parasite mortality and morbidity worldwide and still remains a serious public health problem today. In order to determine the epidemiological profile of amoebae and intestinal amoebiasis in the population of Marrakech. This is a prospective study on the results of parasitological examinations of stool (EPS) performed in adults and children in the department of Parasitology Mycology at the Military Hospital Avicenne (HMA) of Marrakech over a period of two months from 1 May 2018 to 30 June 2018. 70 parasitological examinations of stool (EPS) were performed. Of the 38 positive samples $71.05 \%$ were male with a sex M / F ratio of 2.08. Each patient received at least one EPS, including fresh reading, after Lugol staining, and finally after concentration by the Willis and Ritchie technique. Among the parasites encountered, after identification of the species, the percentage of Blastocystis hominis is $45.76 \%$ followed by Entamoeba coli $35.59 \%$, followed by Chilomastix mesnili $10.17 \%$ then Entamoeba histolytica histolytica and Entamoeba histolytica minuta, the latter having the same proportion 3.39\% and in late Giardia intestinalis with a low proportion of 1.69\%. Amoeba remains, even today, a real public health problem. For example, broadening the scope of these studies by similar surveys focusing on the rural environment would be desirable.
\end{abstract}

Keywords: Amoebae, Entamoeba Histolytica, Entamoeba Dispar, Intestinal Amebiasis, Hygiene, Morocco

\section{Introduction}

The intestinal amoebae are protozoans constituting the subphylum rhizopods and characterized by cell motility through pseudopodia and asexual reproduction. They are classified according to the morphology of the nucleus in two groups: the nucleus type "Entamibe" present in amoebae of the genus Entamoeba and type "Limax" in amoebae of the genus Pseudolimax and Endolimax. The genus Entamoeba gathers several species of which only one is pathogenic certain, because of its necrotic action on the cells, responsible for amebiasis (current name amoebose). According to WHO, this predominantly intestinal parasitosis is the state in which the human organism hosts, with or without clinical manifestations, a protozoan of the rhizopod class Entamoeba histolytica. The latter has long been considered as an infectious agent of variable virulence because of the important difference between the number of healthy carriers and the number of cases of amoebiasis [1].

Amoebiasis is a cosmopolitan parasitosis. About $10 \%$ of the world's population is infected with parasitic amoebae. $10 \%$ of the parasitized subjects have an intestinal or extraintestinal invasive form and of which $90 \%$ are asymptomatic. Amoebiasis is the third leading cause of death by parasitic diseases in the world after malaria and schistosomiasis. It affects approximately 50 million people, of whom 40,000 to 110,000 die each year [2]. It constitutes a real public health problem mainly because of the favorable climatic conditions, the absence or insufficiency of hygiene and sanitation measures most often linked to poverty. These factors which contribute to the perpetuation of the transmission of intestinal parasitoses remain very diverse and complex. Although not pathogenic, all other species of amoeba have an undeniable epidemiological interest, because they are considered 
indicators of orofecal contamination and low levels of hygiene of the carrier subject.

Optical microscopy, during a stool parasitology examination, makes it possible to differentiate specifically the different species of amoebae as well as several other parasitic species. However, mere morphological study may be insufficient; in particular, the differentiation, in nonhematophagous cystic and trophozoite forms, of the pathogenic species: E. histolityca and non-pathogenic: $E$. dispar, which were long confused [3].

The differentiation between E. histolytica and dispar, recommended by $\mathrm{WHO}$, has considerable epidemiological, diagnostic and therapeutic implications. Indeed, E. dispar would be 10 times more common than invasive $E$. histolytica that requires specific treatment [4].

In recent years, several enzymatic, molecular and genetic techniques have been developed to complement the parasitological examination of feces and improve the detection of E. histolytica [5].

The objective of our study is to estimate the prevalence of pathogenic and non-pathogenic intestinal amoebae at the service of mycology parasitology of the Avicenne military hospital of Marrakech (HMA) to assess the degree of orofecal contamination, to study poly-parasitism general and that of amoebas specifically.

\section{Materials and Methods}

\subsection{Type, Place and Duration of Study}

This is a prospective study on the results of stool parasitological examinations (EPS) performed in adults in the department of Parasitology Mycology at the Avicenne Military Hospital (HMA) of Marrakech over a period of two months ranging from 1 May 2018 to 30 June 2018.

\subsection{Patients}

The patients recruited in our study associate adults (military and their families) hospitalized or consultants at the Avicenne Military Hospital (HMA) of Marrakech and the civilian patients addressed hospitals Mohamed VI or the private sector.

\subsection{Methodology}

\subsubsection{Data Collection}

The collection of information is made from the binders and registers of the mycology parasitology department of the Avicenne Military Hospital of Marrakech (HMA). The records of exploitation included: the identity of the patients (name, first name, sex and age), the date of the analysis, the service and the macroscopic and microscopic results of the parasitological examinations of the saddles (EPS).

\subsubsection{Parasitological Examination of Stool}

Each patient received a sterile jar to pick up his morning saddle from the day of the exam. Patients referred to emergency departments defecated in mycology parasitology department.

The stool collected is examined as soon as possible after its emission, first macroscopically to note the appearance, consistency, color and possible presence of blood, mucus or adult parasitic forms.

Stool is also carefully examined microscopically in the fresh state $(0.9 \%$ saline solution) after staining with $2 \%$ Lugol or Merthiolate Iodine Formol (MIF) and after concentration by different techniques. two of which are commonly prepared and used in the HMA parasitology department in Marrakech: the simplified RITCHIE method and WILLIS.

\section{Results}

\subsection{The Characteristics of the Population}

During two months, 70 parasitological examinations of the stool (EPS) were received.

The sex ratio is 2.08 (Figure 1).

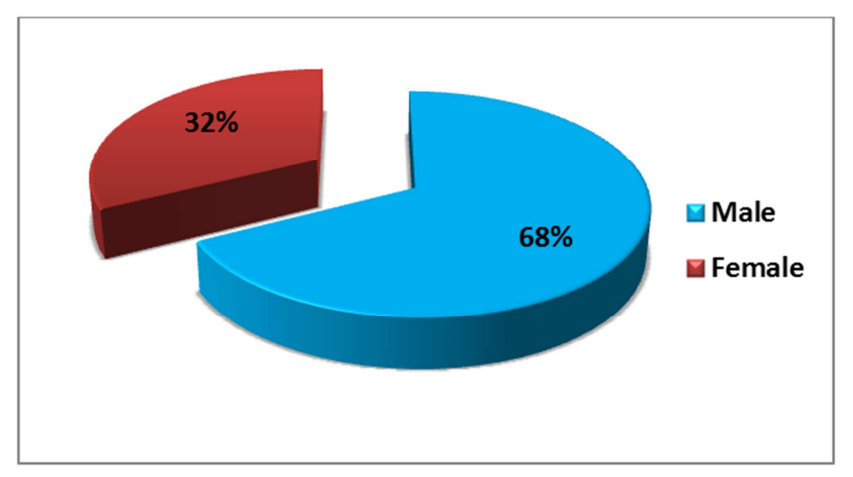

Figure 1. Distribution of the study population by sex.

\subsection{Distribution of Parasitic Species According to the Sex of the Patients}

Le pourcentage des espèces parasitaires isolées enregistré chez les hommes est presque le double que celui chez les femmes pour le cas d'Entamoeba coliet de Blastocystis hominis. Ce pourcentage est plus de 5 fois chez les hommes que chez les femmes pour Chilomastix mesnili. De même aucune femme n'a été parasité ni par Entamoeba histolytica histolytica ni par Giardia intestinalis. 


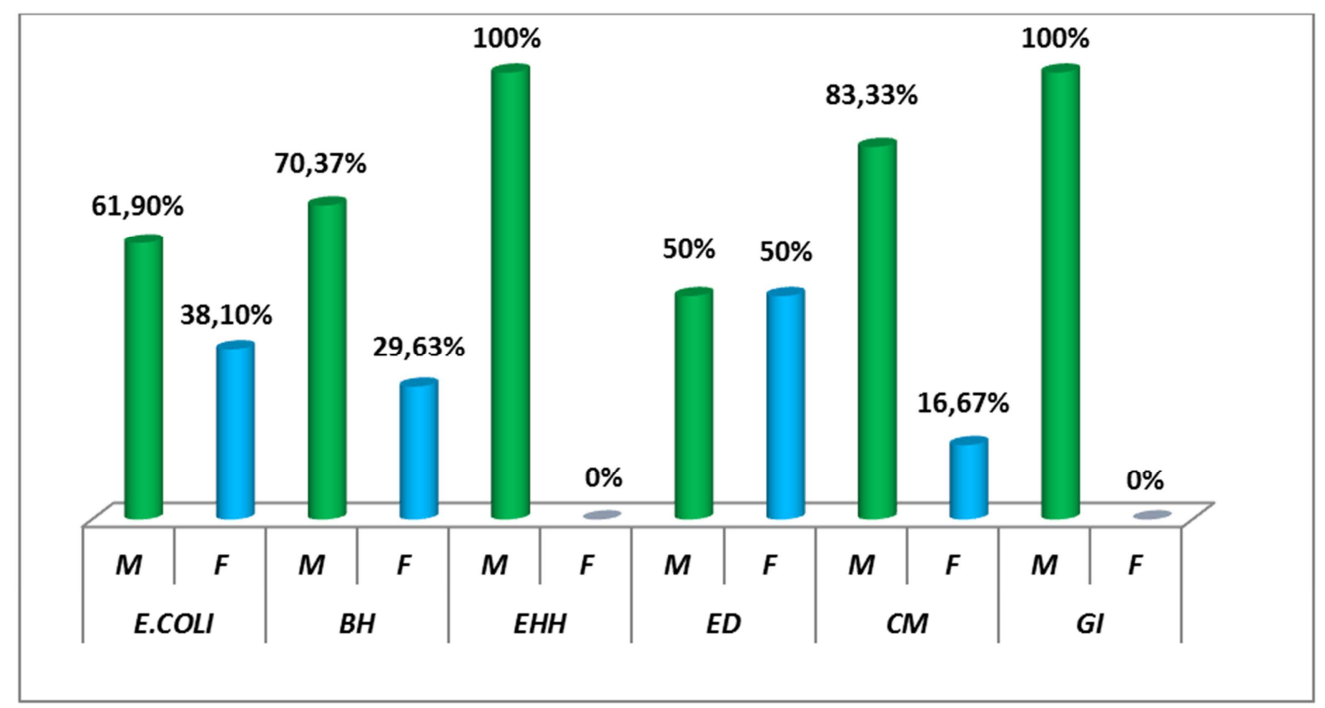

Figure 2. Distribution of the isolated parasite species by sex.

M: Male F: Female E. Coli: Entamoeba coli

BH: Blastocystis hominis EHH: Entamoeba histolytica histolytica

ED: Entamoeba dispar CM: Chilomastix mesnili GI: Giardia intestinalis

\subsection{Identification of the Isolated Parasite Species}

Parasitological examination of stool was used to isolate 5 parasite species, of which Blastocystis hominis is the most common (45.76\%), followed by Entamoeba coli $(35.59 \%)$

and Chilomastix mesnili (10.17\%). Entamoeba histolytica histolytica and Entamoeba histolytica minuta and Giardia intestinalis were only weakly identified with proportions of $3.39 \%$ and $3.39 \%$ and $1.69 \%$ respectively (Figure 3 ).

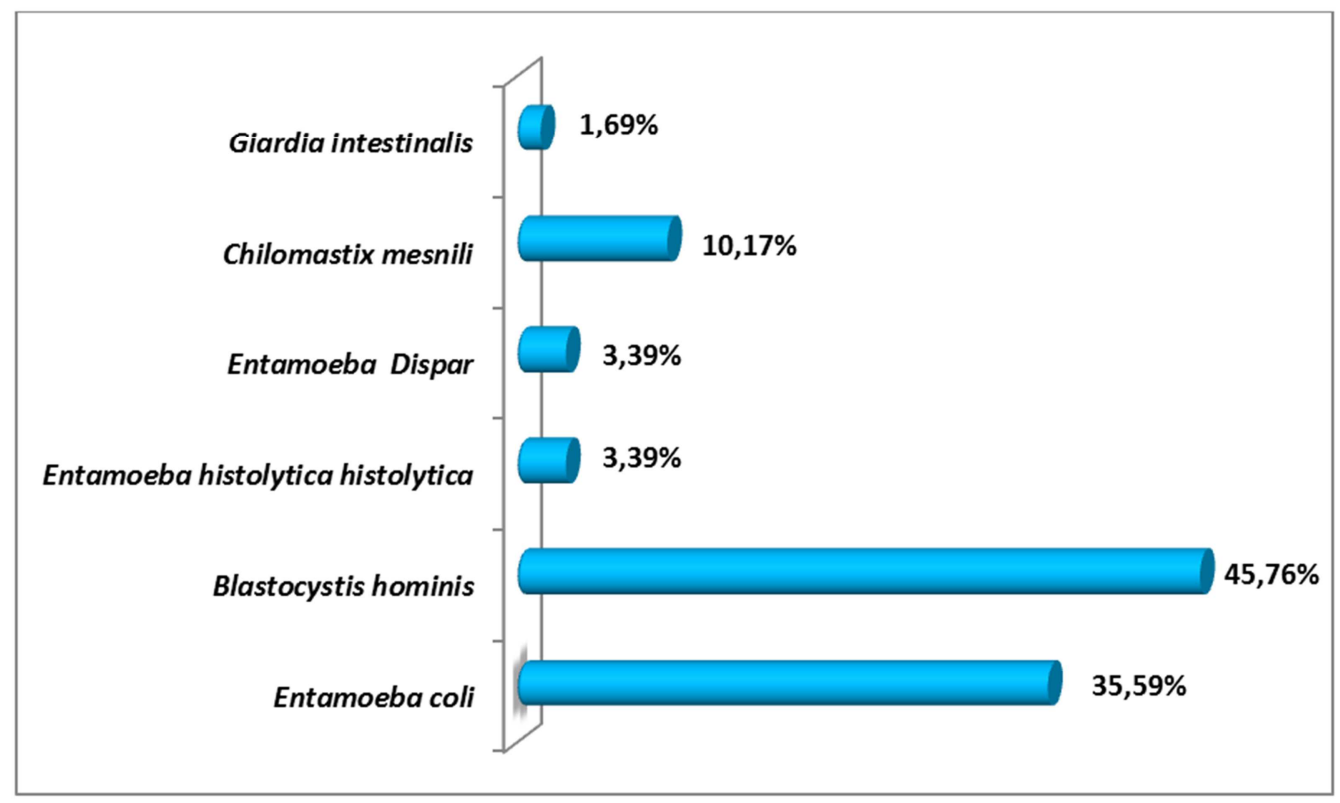

Figure 3. Distribution of isolated parasitic species.

\subsection{Distribution of Species According to the Type of Parasitism}

The analysis of the results according to the parasitism association mode shows that $47.36 \%$ of the cases are in mono-parasitism mode, while $52.64 \%$ of the subjects presents more than one parasite (Table 3).

In the case of poly-parasitism, there are two types of Table 1. Distribution of species by parasitism.

\begin{tabular}{lll}
\hline Mono-parasitism & Poly-parasitism & \\
\hline $47.36 \%$ & $52.64 \%$ & \\
18 & 20 & 3 parasites \\
& 2 parasites & 1 \\
& 19 & $5 \%$ \\
\hline
\end{tabular}
parasite association: 
Figure 4 show the different percentages of the most dominant association (95\%) that of the two parasites. The Blastocystis hominisand Entamoeba coli association is most dominatwith a percentage of more than $85.71 \%$.

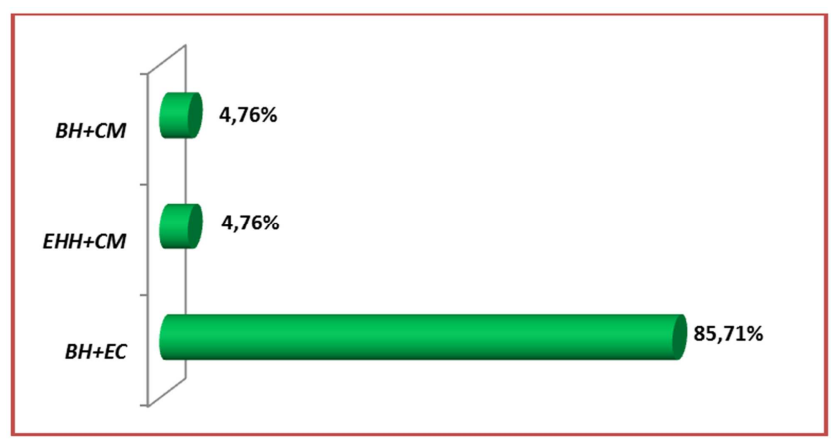

Figure 4. Type of associations observed.

BH: Blastocystis hominis EHH: Entamoeba histolytica histolytica ED: Entamoeba dispar CM: Chilomastix mesniliEC: Entamoeba coli

\section{Discussion}

Our study was based on a sample of 70 samples, consisting of 50 men and 24 women.

The results showed that $51.35 \%$ of the samples analyzed at least one intestinal parasite, this percentage being higher than that found in Morocco, and in Algeria with prevalences of $14.15 \%$ and $35.06 \%$ respectively [6, 7] (Table 2). This difference can be explained by the fact that the other studies were carried out over a wider period (between 01 and 10 years) and a larger sample (between 358 and 4285) [7,6].

However, the prevalence found in this study is lower than that reported in Burkina Faso with prevalences of $54.7 \%$ and $71.05 \%$ respectively $[8,9]$. This is probably due to geographical distribution where it is noted that countries of southern Africa are the most affected because of several factors including hygienic factorsadverse effects and socioeconomic factors. Likewise, the numbers on which the studies are based and also the methodology used to collect the data was different.

Table 2. The prevalence of intestinal parasitism.

\begin{tabular}{lllll}
\hline Our study & Maroc 2009 EL Guamri & Algérie 2016 EL Kassmi & Burkuna Faso 2011 Cissé et al & Burkuba Faso 2014 Zida et al \\
\hline $51.35 \%$ & $14.15 \%$ & $35.06 \%$ & $54.70 \%$ & $71.05 \%$ \\
\hline
\end{tabular}

Regarding the prevalence of amoebae, the study estimated $33.78 \%$ of the subjects infected by amoebae, this frequency is higher than that in Burkina Faso and Algeria with prevalences of $29.8 \%$ and $29.5 \%$ respectively [8,7].

On the other hand, it is lower than that reported in Burkina Faso and Morocco with proportions of $66.71 \%$ and $47.04 \%$ respectively $[6,9,10]$ (Table 3$)$.
The inequality of the prevalence of amoebae in these studies is always related to the geographical difference, moreover the case of Burkina Faso in the year of the study, this can be explained by the rise in temperature during this year. study, knowing that amoebae resist much better in the cold climate than in the hot climate which is an unfavorable condition for the maintenance of their life cycle.

Table 3. The prevalence of amoebae.

\begin{tabular}{lllll}
\hline Our study & Maroc 2009 EL Guamri & Algérie 2016 EL Kassmi & Burkuna Faso 2011 Cissé et al & Burkuba Faso 2014 Zida et al \\
\hline $33.78 \%$ & $47.04 \%$ & $29.5 \%$ & $29.8 \%$ & $66.71 \%$ \\
\hline
\end{tabular}

After khi-two test, the present study showed that the difference is not significant, so patients are at the same risk of infestation regardless of gender. This can be explained by the nature of the population studied which is mainly military, and also by the fact that men are in contact with agricultural fields more than women and less hygiene compared to them.

After identification of the species, the percentage of Blastocystis hominis is $45.76 \%$ followed by Entamoeba coli
$35.59 \%$ followed by Chilomastix mesnili $10.17 \%$ then Entamoeba histolytica histolytica and Entamoeba histolytica minuta, the latter having the same proportion $3.39 \%$ and in late Giardia intestinalis with a low proportion of $1.69 \%$.

The low rate of the pathogenic species Entamoeba histolytica histolytica could be explained by the improvement of the standard of living and hygiene of our population (Table $4)$.

Table 4. The prevalence of parasitic species.

\begin{tabular}{llll}
\hline & Our study & Algérie 2016 EL Kassmi & Burkuna Faso 2011 Cissé et al \\
\hline Entamoeba Coli & $35.59 \%$ & $7.70 \%$ & - \\
Entamoeba Histolytica Histolytica & $3.39 \%$ & $5.60 \%$ & - \\
Entamoeba Histolytica Minuta & $3.39 \%$ & - & $10.40 \%$ \\
\hline
\end{tabular}

In this study, $47.36 \%$ of the subjects presented a monoparasitism and $52.64 \%$ a poly-parasitism. These results differ with those in Algeria where mono-parasitism represents a percentage of $85 \%$ and $15 \%$ of subjects who have poly-parasitism [11] (Table 5).
Table 5. The prevalence of intestinal parasites according to the mode of association.

\begin{tabular}{lll}
\hline & Our study & Algérie 2016 EL Kassmi \\
\hline Mono-parasitism & $47.36 \%$ & $85 \%$ \\
Poly-parasitism & $52.64 \%$ & $15 \%$ \\
\hline
\end{tabular}


This difference between the results can be explained by the duration of the study, which is wide for Algeria and also the studied population which is larger. In the case of polyparasitism, the association of pathogenic and non-pathogenic parasites such as case of Blastocystis hominis which is, according to the studies, probably not pathogenic, but if it exists with a high quantity, gives a strong indication on the existence of pathogenic parasites as the case of association between Blastocystis hominis and Entamoeba histolytica histolytica and Giardia intestinalis $[12,13]$.

The percentages of positivity of the three techniques used (Ritchie and Willis and MIF) are very close and very compatible. All three techniques identified all parasites. But the quality of vision of the parasite differs from one technique to another. In our study, the Willis technique is the best technique to isolate parasites, which corrected the percentage of positivity of $9.25 \%$ followed by Ritchie $(8.65 \%)$ and MIF $(6.98 \%)$, last is the only technique that allows better vision for amoebae.

Many studies showed that Ritchie's technique is a good technique for concentrating the majority of parasitic elements. This method corrected the negativity of direct examination for 12 cases outside of Blastocystis hominis [14].

\section{Conclusion}

Intestinal amoebiasis, this cosmopolitan parasitosis caused by Entamoeba histolytica histolytica, affects according to the who $10 \%$ of the world population of which $10 \%$ of them could be symptomatic.

The present work is a modest contribution to the study of the prevalence of amoeba in the regional military context for a period of time two months. Entamoeba histolytica histolytica and Entamoeba Dispar were only weakly identified with proportions of $3.39 \%$ for each.

This should be interpreted by improving the standard of living and hygiene of the population studied. Population that is predominantly military and urban with a medium socioeconomic level. Similarly, the study period is short enough to have a large staff to confirm the results found. These results do not reflect, the state of resident patients in rural or precarious environments. For example, broadening the scope of these studies by similar surveys focusing on the rural environment would be desirable.

\section{References}

[1] Romain L. Les parasitoses intestinales chez le Macaque Crabier, étude experimentale et recommandations pour le diagnostic et la gestion des Rhizoflagéllés et des Ciliés. Thèse de Doctorat Vétérinaire, Faculté de Medecine de Créteil; 2009.

[2] Anonyme. Amoebiasis. Who Weekly Epidemiol Rec 2007; 72: $97-100$.

[3] Ralston K. S, Petri W. A. Tissue destruction and invasion by Entamoeba histolytica. Trends Parasitol. 2011; 27:254-263.

[4] Mr. Mustapha RABOUA. Épidémiologie des amibes: Expérience du service de Parasitologie de l'Hôpital Militaire Avicenne de Marrakech sur une période de dix ans; 2016.

[5] Fotedar R, Stark D, Beebe N, Marriott D, Ellis J, Harkness J. Laboratory diagnostic techniques for Entamoeba species. Clin Microbiol Rev. 2016; 20(3):511-532.

[6] El Guamri Y, Belghyti D, Achicha A et al. Enquête épidémiologique rétrospective sur les parasitoses intestinales au Centre hospitalier provincial El Idrissi (Kénitra, Maroc) bilan de 10ans (1996-2005).

[7] Mlle KASMI Hadjer et Mlle SAIDOUNI Asma. Etude de la prévalence des protozooses intestinales diagnostiquées au sein $\mathrm{du}$ laboratoire de parasitologie-mycologie du CHU de Tlemcen-mémoire de fin d'étude pour obtention du diplôme de docteur en pharmacie 2016.

[8] Cissé M, Coulibaly SO, Guiguemdé RT. Aspects épidémiologiques des parasitoses intestinales rapportées au Burkina Faso de 2011.

[9] Zida et al. Prévalence du parasitisme intestinal en milieu carcéral à Ouagadougou (Burkina Faso) Médecine et Sante Tropicales 2014.

[10] A. Benouis, Z. Bekkouche, Z. Benmansour. Etude épidémiologique des parasitoses intestinales humaines au niveau du C. H. U. d'Oran. International Journal of Innovation and Applied Studies. 2013; 2: 613-620.

[11] Ralston K. S, Petri W. A. Amoebiasis. Who Weekly Epidemiol Rec 2007; 72: 97- 100.

[12] Ali IK. Intestinal amoebae. Clin Lab Med 2015; 35:393-422.

[13] Belhamri N, MOUTAJ R. Profil épidémiologique des parasitoses intestinales au service de Parasitologie Mycologie à l'Hôpital Militaire Avicenne de Marrakech. Thèse de doctorat en médecine, faculté de médecine et pharmacie Marrakech; 2015.

[14] Gill GV, Beeching NJ. Tropical Medicine. 2004; 5:153-159. 